\title{
Magnetic resonance imaging for congenital lung malformations
}

\author{
Beverley Newman ${ }^{1}$
}

Received: 2 November 2020 / Revised: 6 January 2021 / Accepted: 11 February 2021 / Published online: 10 March 2021

(C) Springer-Verlag GmbH Germany, part of Springer Nature 2021

\begin{abstract}
Congenital lung malformations are most often identified on prenatal US screening. Fetal MRI is often performed to further evaluate these lesions. Although some of these lesions might cause prenatal or early postnatal symptoms that require urgent management, the majority are asymptomatic at birth and might be subtle or invisible on chest radiographs. Postnatal imaging is frequently deferred until 3-6 months of age, when surgery or long-term conservative management is contemplated. High-quality imaging and interpretation is needed to assist with appropriate decision-making. Contrast-enhanced chest CT, typically with angiographic technique, has been the usual postnatal imaging choice. In this review, the author discusses and illustrates the indications and use of postnatal MR imaging for bronchopulmonary malformations as well as some differential diagnoses and the advantages and disadvantages of MR versus CT.
\end{abstract}

Keywords Bronchopulmonary malformation $\cdot$ Infants $\cdot$ Lung $\cdot$ Magnetic resonance imaging $\cdot$ Postnatal

\section{Introduction}

Although multiple associated anomalies are sometimes included in the grouping of congenital lung malformations, this discussion is confined to the five major entities included as the primary bronchopulmonary malformations: bronchogenic cyst, congenital pulmonary airway malformation (CPAM), pulmonary sequestration, congenital lobar overinflation and bronchial atresia. These entities are closely related with differing features including components of foregut, airway, vascular and parenchymal abnormality [1]. The unifying underlying etiology of these lesions is thought to be partial or complete airway obstruction in utero, with resultant dysplastic lung development with features varying depending on the degree of obstruction and timing in utero [2]. Bronchial atresia commonly accompanies all of these entities [3, 4]. Congenital lung malformations are among the most common abnormalities identified in utero, affecting approximately 1 in 2,500 pregnancies [5].

\section{Beverley Newman}

bev.newman@stanford.edu

1 Department of Radiology,

Stanford Children's Hospital at Stanford University,

725 Welch Road, Stanford, CA 94304, USA

\section{Prenatal and early postnatal imaging, management and differential diagnosis}

The majority of bronchopulmonary malformations are first identified on prenatal US screening, most often in the second trimester [5-9]. Many undergo further evaluation in utero with one or more fetal MRI examinations, which have been shown to be accurate for assessing location, size, extent, mass effect and internal features, and for assisting in management decisions such as method, time and location of delivery or occasionally in utero intervention [7, 10-12]. While MRI examination has been shown to be safe during pregnancy, MR contrast agents are generally not used prenatally because they can cross the placenta and their safety for the fetus is uncertain. Many bronchopulmonary malformations decrease in size or conspicuity or even disappear during the 3rd trimester MRI $[9,13]$; however, they may remain unchanged in size or occasionally enlarge. In utero hydrops fetalis can result from obstructed venous return related to mass effect and midline shift by the lesion, necessitating emergent treatment or delivery $[5,10,12,14]$.

Several characteristic imaging features of bronchopulmonary malformations are evident on both pre- and postnatal imaging $[6$, $7,9,15-17]$.

1) Macroscopic cysts. This is a typical feature of bronchogenic cyst (usually single or multilocular cyst) and CPAM, multiple clustered small $(0.5-$ to $2-\mathrm{cm})$ or large cysts (largest cyst $>2 \mathrm{~cm}$ ). 
2) Systemic arterial supply to affected lung, a primary feature of pulmonary sequestration.

3) Mucoid impaction, branching intraluminally in airway distal to more central bronchial atresia.

4) Focal overinflated lung, a feature of lobar/segmental overinflation distal to obstructed or atretic bronchus; it is fluidfilled prenatally and gradually air-filled postnatally.

5) Hybrid lesions. These have multiple features, often a mix of CPAM, pulmonary sequestration and bronchial atresia.

Large or symptomatic bronchopulmonary malformations at birth might undergo urgent postnatal imaging, usually plain radiographs, US or $\mathrm{CT}$, and they might require emergency surgical management [6, 18]. However, the majority of lesions are asymptomatic at birth; they might be evident, not visible, or exhibit subtle findings on chest radiographs $[10,12,14]$. Early postnatal US might be helpful in confirming the presence, size and type of a known prenatal lesion, especially if the malformation is not yet aerated [17]. Most asymptomatic lesions that were well characterized in utero can have postnatal cross-sectional imaging deferred until 3-6 months of age, when surgical removal versus long-term conservative management is being contemplated. Imaging most often consists of contrast-enhanced CT angiography to evaluate/confirm the type, size, location, extent and vascular supply/drainage of the lesion, and relationship to the airway and adjacent structures $[8,11,17,19]$. Benefits of delaying postnatal imaging beyond the newborn period include improvement in renal function and more accurate characterization by allowing time for lesions to become aerated because frequently prolonged retention of fetal lung fluid is associated with abnormal or obstructed airways.

It is important to accurately assess and describe the features of a congenital lung lesion. A small lesion, presence of bronchial atresia (mucoid impaction) and focal overinflation are features that might support nonsurgical management, whereas a large lesion, large systemic arterial supply and multi-cystic features are more likely to necessitate surgical resection [10]. Surgery is often performed using minimally invasive techniques (thoracoscopy) and typically consists of lobectomy. Segmental resection is more difficult because the surgeon frequently cannot define the margins of the lesion from normal lung. Reasons to justify surgical resection include the risk of infection, although the incidence is not definitively known but thought to be small, and the small concern for current or future malignancy $[5,6,12,18]$.

Other lesions might mimic or need to be differentiated from bronchopulmonary malformations, both pre- and postnatally. Bronchopulmonary malformations generally have a typical imaging appearances prenatally (T2-weighted hyperintense signal with or without small or large cysts, systemic vessels, mucoid impaction) [15, 20]. Other chest and mediastinal masses including diaphragmatic hernia and neurogenic and vascular lesions are usually readily distinguished from bronchopulmonary malformations on imaging. However, differentiation from other rare chest neoplasms, especially cystic pleuropulmonary blastoma (PPB), can be more difficult. Lung neoplasms are almost never identified before the 3rd trimester, as opposed to

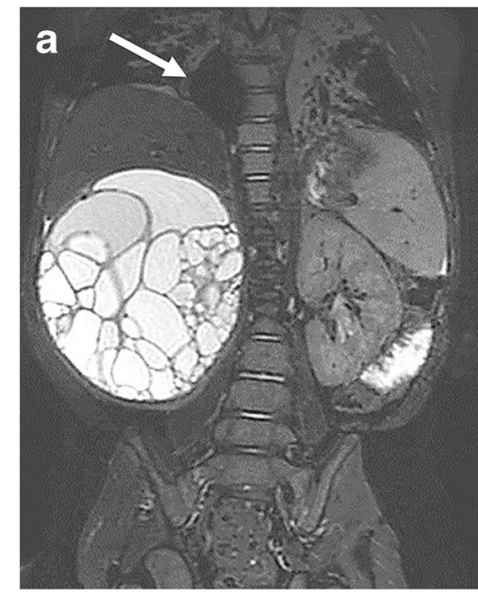

Fig. 1 Cystic pleuropulmonary blastoma (PPB) in a 19-month-old boy with no abnormal prenatal history. He had MRI for an abdominal mass, with an incidental finding of lower right lung cyst. a Coronal T2-weighted MRI shows a large multiloculated cystic mass in the right kidney, likely a multilocular cystic nephroma. There is an incidental finding of a prominent air-filled cyst in the medial right lower lobe (arrow). Note the anesthesiarelated atelectasis at the lung bases, left greater than right. These findings raised concerns that the chest lesion might be cystic pleuropulmonary blastoma, which can be associated with other tumors including multilocular cystic nephroma. In many cases there is an associated DICERI genetic mutation (found later to be present in this child). b Subsequent chest CT

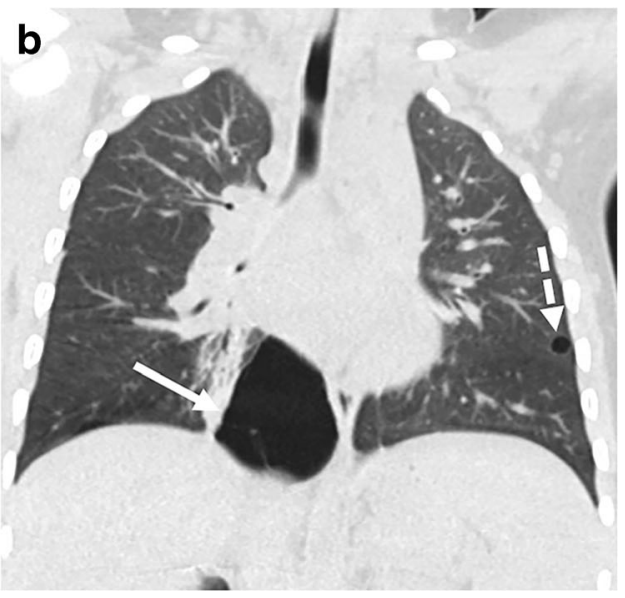

scan, coronal reconstruction, shows a large medial right-lower-lobe cyst (solid arrow), as seen on earlier MRI. There are also several smaller scattered intrapulmonary cysts, one of which is shown on the left (dashed arrow). The most likely diagnosis was PPB. The lack of prenatal history of a cystic lung lesion, peripheral location, multiple cystic foci and association with DICER1 mutation and another neoplasm all served to differentiate this case from congenital pulmonary airway malformation. The large right lower lobe cyst was resected and confirmed pathologically as type 1 cystic PPB. The other cysts were to be followed clinically and by imaging. These could grow and evolve into a more aggressive type of PPB; however, they could also become quiescent cysts 
bronchopulmonary malformations, which are usually identified in the 2nd trimester [9]. Solid neoplastic lesions such as infantile fibrosarcoma and peribronchial myofibroblastic tumor and chest wall mesenchymal hamartoma are usually different in their imaging appearance as compared to bronchopulmonary malformations (more heterogeneous, can be iso- to hypointense on T2-weighted images). Occasionally bronchopulmonary malformations have an atypical appearance with $\mathrm{T} 2$-weighted hypointensity, possibly caused by myxoid or immature highly cellular mesenchymal components [9, 20-22]. Fetal lung interstitial tumor is a rare benign lesion that is uniformly T2weighted hyperintense on MR [9]. It is considered unlikely that bronchopulmonary malformations such as CPAM evolve into neoplasms like PPB; rather, it is thought that lesions that are ultimately diagnosed or recur as PPB were probably mischaracterized initially [5]. There might be a small association between pre-existing congenital lung cysts, especially large cyst CPAM, and development of broncho-alveolar carcinoma in adults, but any association is likely insufficient to justify surgical resection of all lesions [5]. Prenatally, US and MRI are the imaging modalities employed for suspected nonbronchopulmonary malformation chest masses, whereas CT is most often the primary postnatal choice $[6-8,23]$. While differentiating CPAM from cystic PPB on imaging alone in an individual lesion can be difficult, the combination of prenatal and postnatal history, lesion location, multiplicity and associated anomalies helps to separate these lesions. Cystic PPB is usually found in young infancy, occasionally late pregnancy; it more often presents with pneumothorax (peripheral lesion); it more often has complex and multiple cystic foci; and it is associated with a DICER1 mutation with a family history of neoplasms and propensity for concurrent or subsequent neoplasms, especially thyroid, renal (multilocular cystic nephroma) and gonadal lesions (Fig. 1) [6, 9, 12, 14, 18, 21]. Prenatal detection, systemic feeding vessel, mucoid impaction, and asymptomatic and overinflated lung favor the diagnosis of CPAM or other bronchopulmonary malformation $[9,18]$.

\section{Postnatal magnetic resonance imaging for congenital lung lesions: techniques and indications}

A closely applied multi-array coil covering the chest is optimal for MR imaging of suspected lung lesions. Typical MR imaging protocols for evaluating congenital lung lesions postnatally have
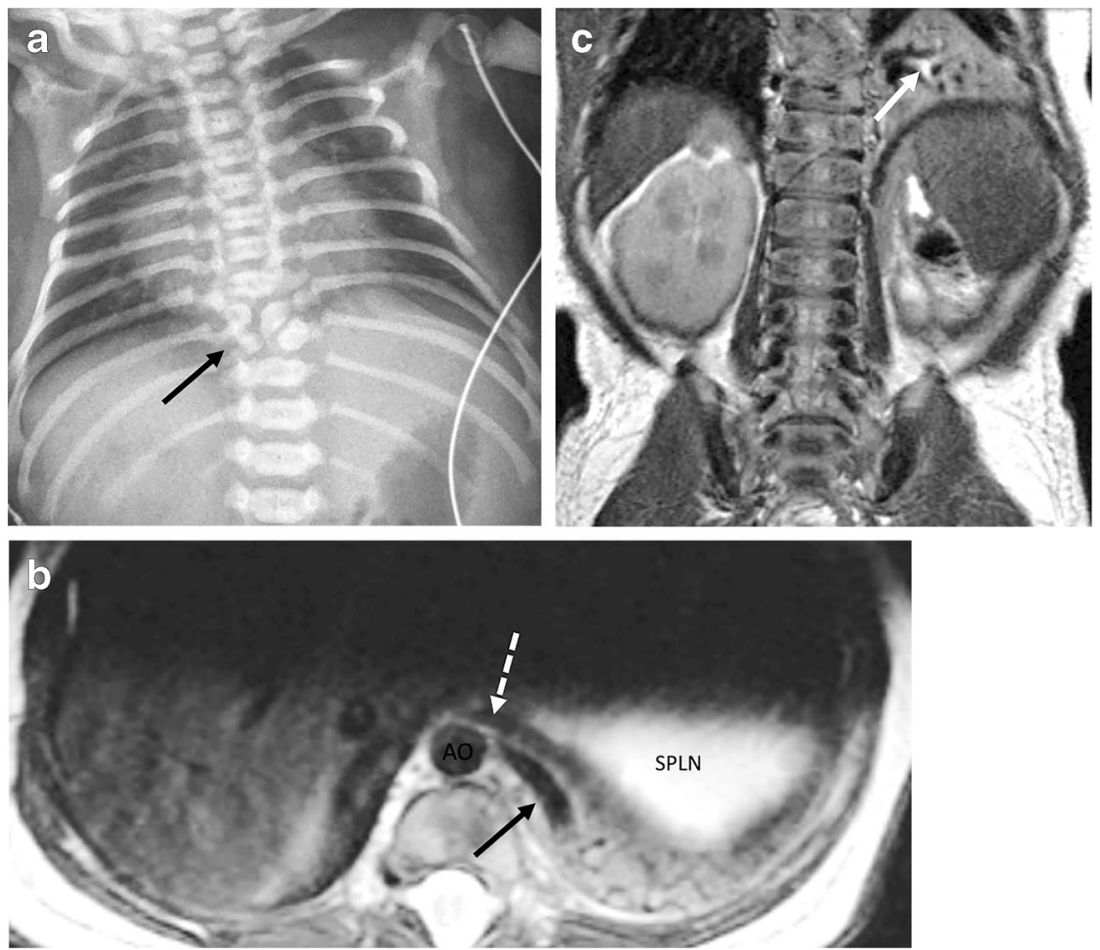

Fig. 2 Imaging in a 13-day-old boy with known left-lower-lobe lesion prenatally and incidental imaging of pulmonary sequestration on a spine MRI. a Anteroposterior chest radiograph shows an ill-defined medial leftlower-lobe density. T9 vertebral anomaly (arrow) led to spine MR for evaluation of possible associated cord abnormality. b Axial T2-weighted MR image of lung bases from spine MR shows anterior saturation band to enhance spine imaging. There is a nonaerated, moderately vascular and somewhat T2bright lesion in the posteromedial left lower lobe, with systemic arterial supply (dark-blood sequence) from the lower thoracic aorta (solid arrow) and venous drainage toward a systemic vein (dashed arrow), consistent with an extralobar pulmonary sequestration. SPLN spleen. c Coronal T2-weighted image from spine MR demonstrates the triangular medial left-lower-lobe nonaerated sequestration. In addition to central dark branching vessels, there is a bright central branching structure (arrow) within the sequestration from mucoid impaction in bronchi, consistent with associated bronchial atresia. Note the absence of the left kidney, with bowel in the left renal fossa 

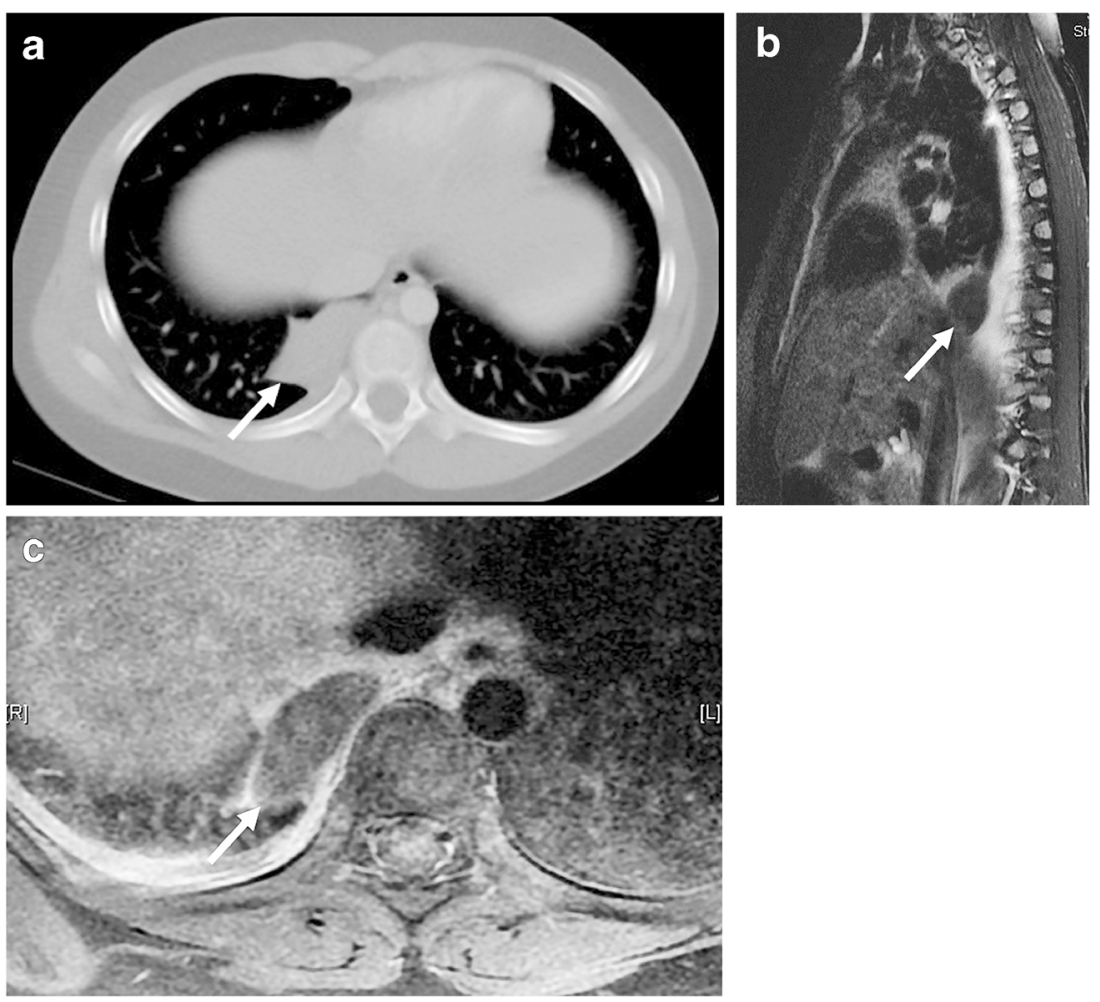

Fig. 3 Incidental chest mass in a 12 -year-old boy presenting with back and abdominal pain. a Contrast-enhanced CT scan for appendicitis (negative abdomen), axial slice at the lung bases, shows a poorly enhancing mass (arrow) in the right lower lobe versus posterior mediastinum. There is a small right pleural effusion. b Sagittal T2-W MR obtained to further evaluate the right-side mass shows an oval T2hypointense mass in the right lower lung posteriorly (arrow), surrounded by T2-hyperintense pleural fluid. c Axial T1-weighted post-contrast MRI of the right lung base shows that the posteromedial oval right-lower-lobe

included fast T1-weighted and T2-weighted sequences in axial, coronal and occasionally sagittal planes. Sometimes diffusionweighted sequences are added, usually in the axial plane. Sequences employed include: two-dimensional (2-D) steadystate free precession; single-shot fast spin echo; respiratorygated proton-density and T1- and T2-weighted fast spin echo; and fast spoiled gradient echo acquisition or 3-D volume T1weighted inversion pulse sequence with fat suppression, which can be obtained both pre- and post-contrast and reconstructed into fat, water and in- and out-of-phase images. Dynamic multiphasic angiographic sequences include 3-D gradient echo fatsuppressed respiratory/cardiac-gated sequences or time-resolved MR angiography $[12,16]$. A more recent addition has been the development of ultrashort echo-time T1-weighted sequences (both pre- and post-contrast), which greatly improve the visibility of lung parenchyma.

Magnetic resonance chest imaging studies typically take approximately $45 \mathrm{~min}$ to $1 \mathrm{~h}$ to complete [16]. Children undergoing these MR examinations usually require sedation or anesthesia. Although intubation or breath-holds might not be required for imaging, sedation/anesthesia is frequently associated with substantial lung atelectasis that can obscure intrapulmonary lesions. mass (arrow) has minimal peripheral enhancement. Note that the pleural fluid is T1-hyperintense, suggesting blood (containing methemoglobin and protein-rich plasma) or other protein-rich fluid with T1-shortening effects. The boy's pain symptoms, location and appearance of the lesion with absent perfusion as well as T1/T2 bright pleural fluid suggested the diagnosis of a torsed, infarcted extralobar pulmonary sequestration with hemorrhagic pleural effusion. This was subsequently confirmed surgically and on pathology

As with CT imaging, if needed, intubation/airway recruitment techniques can be employed to mitigate atelectasis [24]. Prone imaging is not reliably helpful in mitigating anesthesia-related atelectasis and is more difficult to achieve, with increased safety concerns in an anesthetized child [24]. MR safety is very important. All equipment must be MR-compatible, and children, parents and accompanying personnel need to be carefully screened before entering the scanner area [23].

When searching for cases of bronchopulmonary malformations or similar lung masses imaged on MR rather than or in addition to CT, "we found that the reasons for MR imaging of lesions at our institution fell into several major categories that sometimes overlapped."

1) Imaging for another reason with incidental visualization of a lesion. Examples included MR imaging of the spine for suspected mass/anomaly and MRI or CT of the chest or abdomen for other reasons with incidental lung finding. In this context it is important to be able to recognize and characterize the pulmonary lesion, although imaging sequences and coverage might not have been ideal (Figs. 1, 2 and 3). 

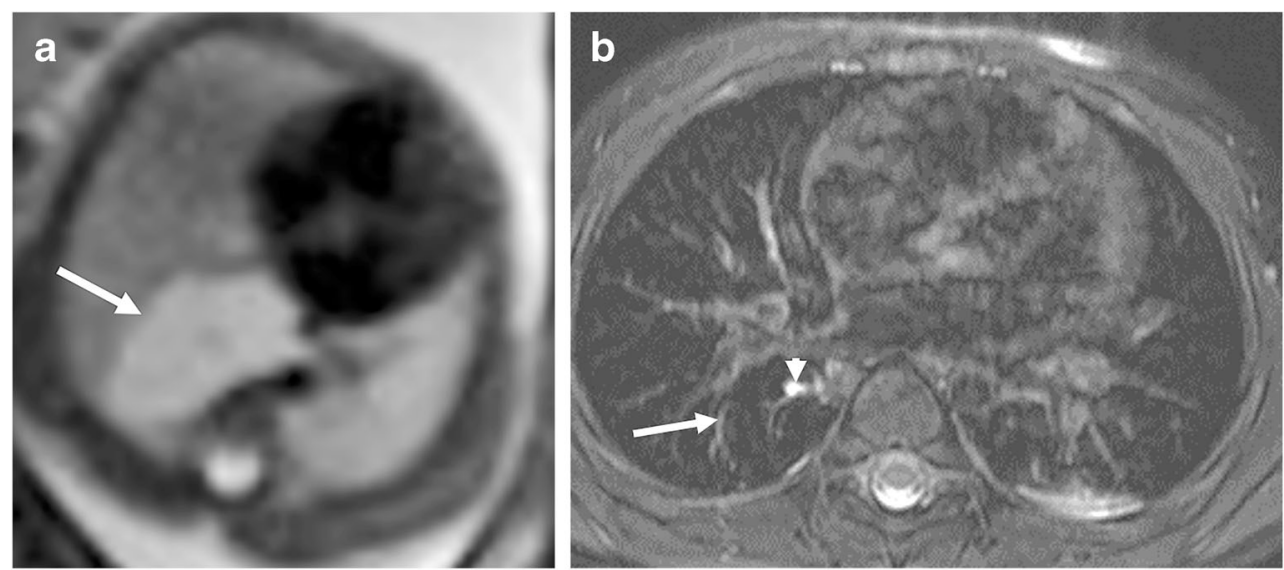

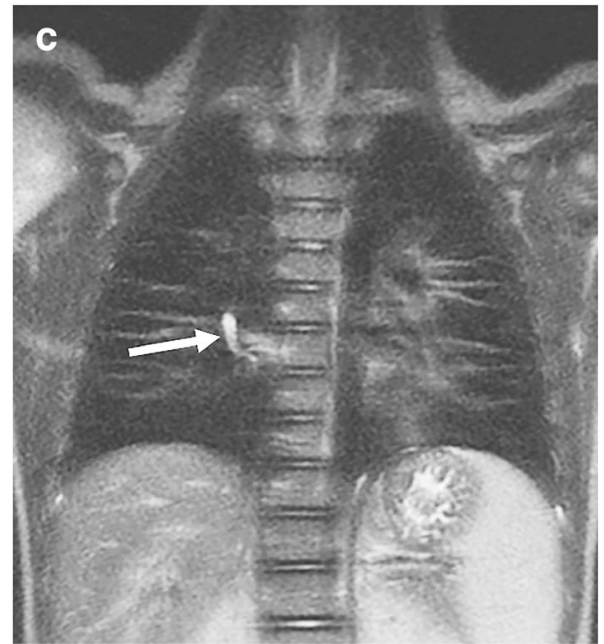

Fig. 4 Right-lower-lobe lesion identified in utero and then lost to followup postnatally until 2 years of age in a girl. a Axial T2-weighted prenatal MRI slice at 22 weeks of gestation. Segmental homogeneous T2hyperintense right-lower-lobe mass (arrow) was thought to most likely represent segmental lung overinflation. The lesion was much smaller and less T2-bright on a follow-up fetal MR at 37 weeks of gestation (not shown). b The girl had MRI at 2 years old (parents preferred to avoid $\mathrm{CT}$ radiation exposure). Axial T2-weighted image shows a posteromedial right-lower-lobe lesion (long arrow) with central hyperintense mucoid impaction (short arrow) and surrounding air-trapping, consistent with segmental bronchial atresia with overinflation. Note how much brighter

2) Known lesion follow-up. In these cases, there was typically concern related to CT radiation exposure and a parental preference for MR to confirm presence or follow a lesion that had been identified in utero or had prior $\mathrm{CT}$ findings (Figs. 4, 5 and 6) [25].

3) Mass evaluation/differentiation. In these cases, the mass was not specifically suspected to be a bronchopulmonary malformation or imaging was performed to differentiate the mass from a bronchopulmonary malformation in a location that was well visualized on MR, such as paraspinal, mediastinal or even abdominal areas (Figs. 7 and 8).

4) Complex or multiple lesions. These lesions might have been incompletely characterized or had a confusing appearance on CT (Figs. 9, 10 and 11) [21].

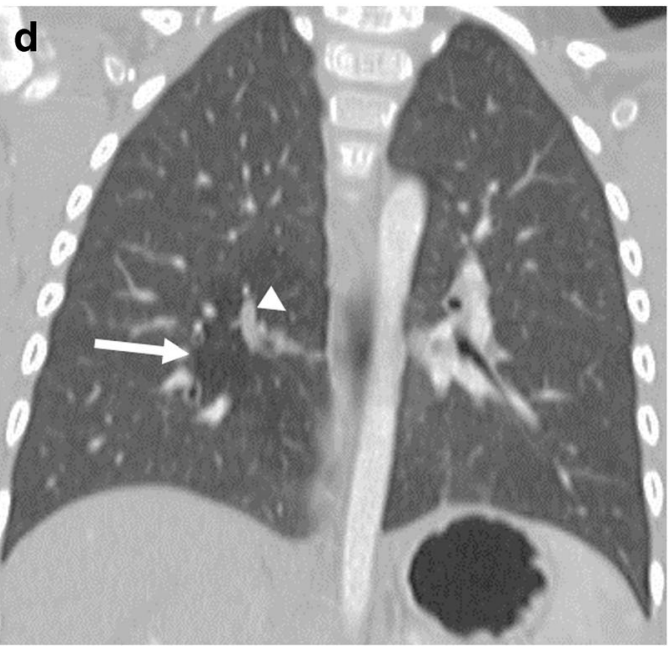

the mucoid impaction is than adjacent vessels. c Coronal T2-W MR image shows T2-bright branching mucoid impaction in the lesion (arrow). This MR was obtained before the availability of ultra-short echo-time imaging of the lung, so the lung detail is relatively poor. d Follow-up coronal contrast-enhanced CT at 3 years of age shows a posteromedial segmental hyperlucency (arrow) with central mucoid impaction (arrowhead), again confirming segmental overinflation with bronchial atresia. The mucoid-impacted bronchus is a little larger but only slightly less dense than vessels and can be difficult to identify on CT. This lesion was not resected

\section{Magnetic resonance imaging for congenital lung lesions: strengths/weaknesses, advantages/disadvantages}

Magnetic resonance is effective in imaging congenital lung lesions [16], making it generally easy to differentiate cystic from solid lesions (Figs. 7 and 11). Proteinaceous cyst content is easier to identify on MR, where it might be hyperintense on both T1-weighted and T2-weighted images pre-contrast with only rim enhancement, whereas on a contrast-enhanced CT a hyperdense proteinaceous cyst is more likely to be mistaken for an enhancing solid mass [26]. Mucoid impaction is noticeably hyperintense on T2-weighted imaging (usually T1-weighted hyperintense also) [27] and tends to be easier to identify and 

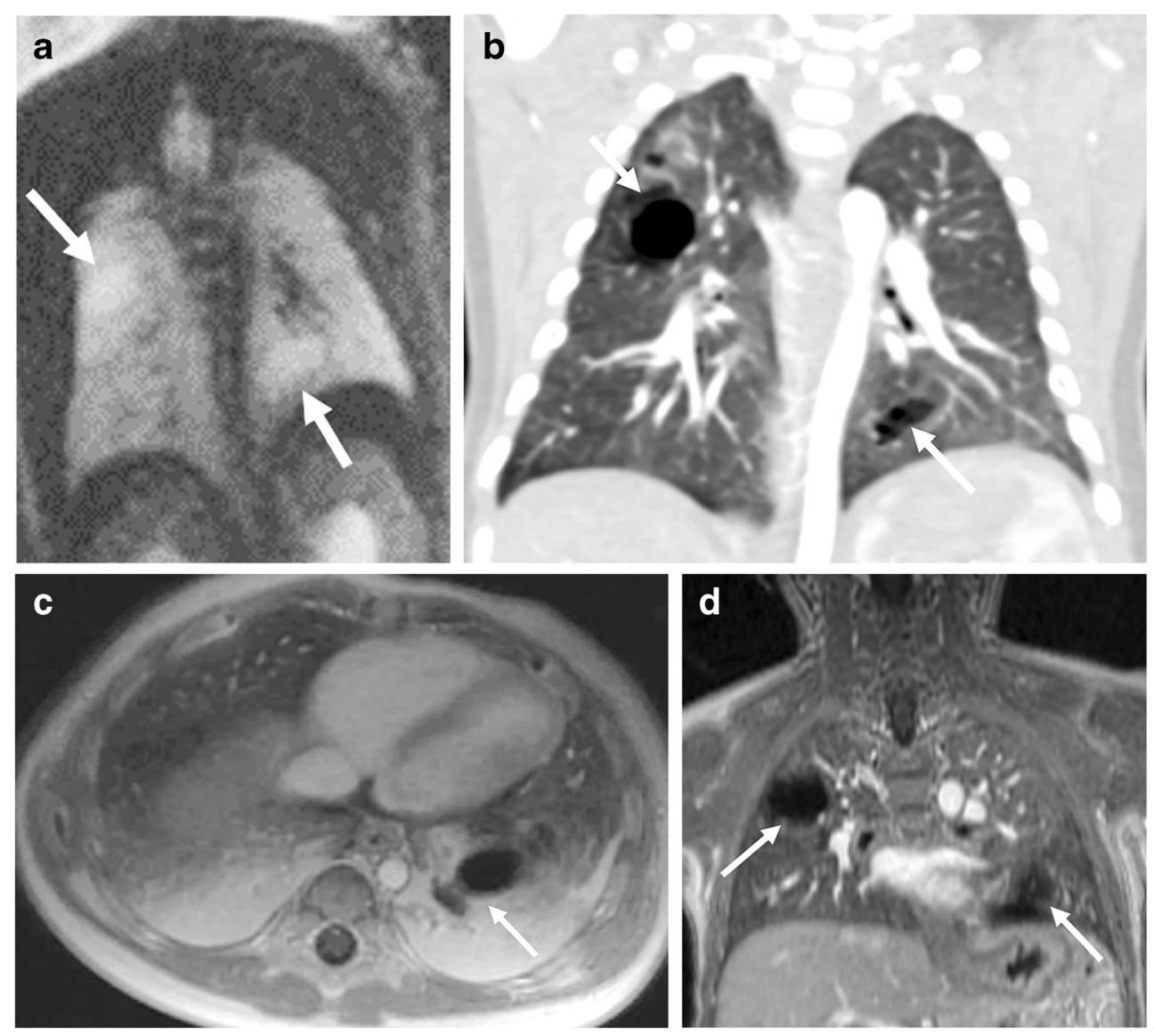

Fig. 5 Follow-up prenatal MR in a fetus with a history of twin-twin transfusion syndrome with laser ablation and fluid amnioreduction, with demise of the smaller donor twin at 22 weeks. a Fetal MRI at 31 weeks of gestation shows multiple T2-hyperintense large cysts in both lungs (arrows), which were not present on the prior MR. b CT scan of the girl at 4 months of age. Coronal reconstruction shows multiple air-filled lung cysts (arrows). c MRI age 10 months (parents did not want repeat CT). Axial ultra-short echo-time T1-weighted post-contrast sequence shows a multilocular air-filled cystic lesion (arrow) at the left lung

separate from vessels on pre-contrast T2-weighted MR (Figs. 2 and 4), as compared to contrast-enhanced CT where the impacted mucus, although slightly lower in attenuation, might be more difficult to distinguish from vessels (Fig. 4). MR and CT angiography both identify aberrant arteries and veins, although with CT angiography, because of radiation concerns, there is generally only one opportunity to optimize vascular opacification, whereas the rapid multiphasic capability of MR is much more forgiving. Mass characterization and evaluation of associated cardiovascular anomalies are as good as - and sometimes better - with MR as compared to CT [23] (Figs. 10 and 11). Kellenberger et al. [16] described similar sensitivity of MR to CT in detecting and characterizing a group of bronchopulmonary malformations. On MR, dynamic enhancement patterns of bronchopulmonary malformations could be assessed, the solid components of bronchopulmonary malformations showed slow perfusion with delayed enhancement compared to normal as well as atelectatic lung, and cystic lesions showed rim enhancement. Arterial supply base, similar to findings on prior CT. There is moderate posteriordependent anesthesia-related atelectasis. d MRI at age 2 years. Coronal T1-W ultra-short echo-time post-contrast sequence shows the air-filled cysts unchanged (arrows). The etiology of these lesions was not entirely clear; they were not resected or biopsied. However, cystic lung lesions have been described in other twin-twin pregnancies that were thought to be most consistent with congenital pulmonary airway malformations on pre- and postnatal imaging [25]

and venous drainage were well identified and mucoid impaction was easily visualized [16]. Vascular supply, as might be expected, is less well visualized on non-contrast MR studies [8].

A major advantage of MR imaging is the lack of exposure to ionizing radiation. In terms of imaging and especially MR angiography and post-contrast imaging, multiple sequences and angiographic phases can be obtained with no cost except time. Additionally, the multiple sequence choices of MRI including T1-weighted, T2-weighted, phase-contrast, 3-D T1 dynamic or 4-D time-resolved MR angiography and diffusion-weighted imaging provide more than anatomy with greater tissue, physiology and vascular flow characterization. MRI also has the potential for greater functional lung assessment including perfusion and ventilation [28]. MRI has inherently superior softtissue contrast, for example in the mediastinum, and better delineation of anatomical detail without intravenous contrast agent [28]. Both MR and CT images can be reconstructed into a large variety of multiplanar and 3-D views to better appreciate 

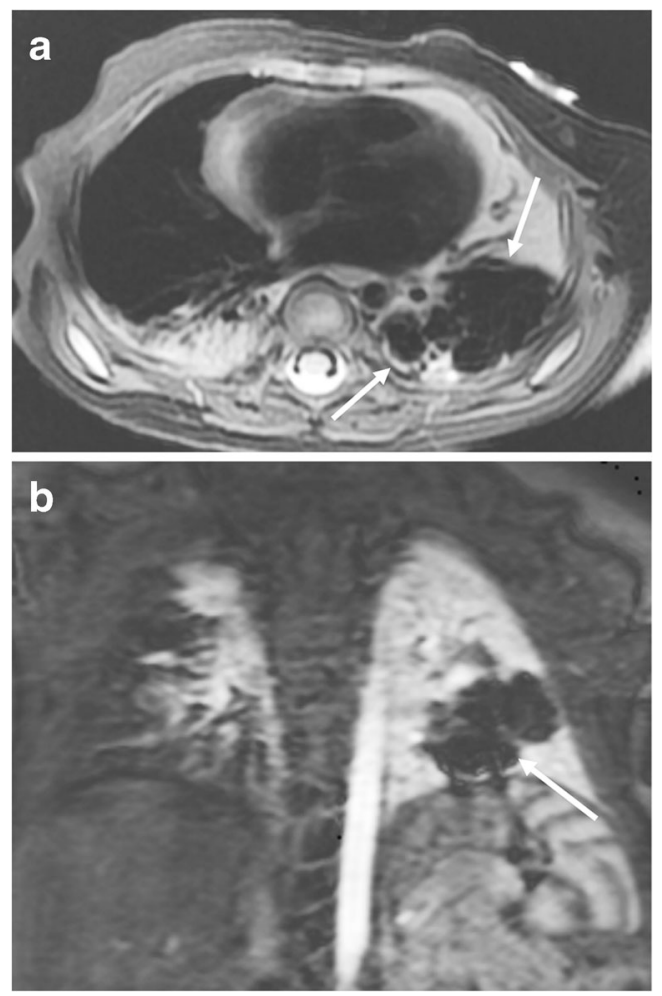

Fig. 6 Follow-up imaging in a 4-month-old girl with a prenatal diagnosis of left-lower-lobe congenital pulmonary airway malformation (CPAM). There was a subtle lower-lobe opacity on chest radiograph at birth (not shown). MRI was obtained at 4 months to confirm whether the lesion was still present. Parents refused CT because of ionizing radiation exposure. a Axial T2-weighted (black-blood) MR sequence shows a multi-cystic airfilled lesion (arrows) in the posterior left lower lobe, consistent with large-cyst CPAM (largest cyst $>2 \mathrm{~cm}$ ). The surrounding normal left lung is atelectatic; there is also right-side-dependent atelectasis. b Coronal T1-weighted post-contrast MR image shows left-lower-lobe aerated large cyst CPAM (arrow). No abnormal systemic arterial supply is seen. The entire remaining left lung is atelectatic from inadvertent right bronchus intubation under anesthesia. The persistent aeration of the CPAM lesion reflects air-trapping from an abnormal airway connection; the phenomenon is similar to the prolonged retention of fluid seen in many bronchopulmonary malformations in the neonatal period. If the lesion were not aerated, the atelectatic lung would likely obscure it. The MR images were considered sufficient for surgical guidance for thoracoscopic resection of the left lower lobe. Diagnosis of large-cyst CPAM was confirmed on pathology

the precise anatomy and relationships of the different structures.

Magnetic resonance imaging also has a number of disadvantages. The many possible imaging pulse sequence choices tend to make these examinations very lengthy, often necessitating general anesthesia in younger children $(<8$ years $)$ [16]. Estimates of severe adverse event perioperative anesthesia risk in children is $\sim 1$ in 10,000 , greater in neonates and children with serious underlying conditions [29]. Considerable attention has been paid recently to potential negative cognitive effects on the developing brain in young children undergoing general anesthesia [29]. The United States Food and Drug Administration has placed warning labels on general anesthetics and some sedative medications that states, "Exposure to these agents for lengthy periods of time or over multiple procedures may negatively affect brain development in children younger than 3 years" $[29,30]$. The need for anesthesia and the length of MR examinations also creates imaging access and scheduling difficulties. Avoiding anesthesia and especially intubation is always desirable but even more so in the time of the current pandemic caused by coronavirus SARS-CoV-2 disease (COVID-19). Intravenous anesthetic medications are an alternative but are also associated with anesthesia-related atelectasis without the ability to use effective airway recruitment techniques [24]. Faster sequences, motion reduction, gating and navigating techniques, contrast agents with a more prolonged intravascular phase, limited targeted studies, effective immobilization, distraction or wrap-and-feed methods in young children have been helpful in allowing for more effective non-sedated MR imaging $[15,28]$ (Figs. 5 and 8).

Additionally, MRI has not been considered a good modality for imaging parenchymal lung lesions because of the intrinsic low MR signal from proton-poor lung tissue. The relatively recent development of robust ultrashort echo time MR imaging with radial, spiral or conical 3-D k-space trajectories (Fig. 5) has greatly improved visualization of the lung on MRI, markedly mitigating this concern [28]. Some of these techniques appear robust in the face of flow and motion allowing for free-breathing imaging [28]. MRI, however, still appears to show less detail than CT of smaller lung lesions $<5 \mathrm{~mm}$ [27].

Another disadvantage of MRI is the use of gadolinium chelate contrast agents. Problems include the small possibility of nephrogenic systemic fibrosis in children with renal dysfunction; however, the occurrence of this in small children is negligible. There are also concerns related to gadolinium deposition in the brain with unknown long-term effects. This appears to be most marked with linear gadolinium chelate agents, which have been replaced by macrocyclic agents at many centers [16].

\section{Computed tomography vs. magnetic resonance for congenital lung lesions}

Magnetic resonance imaging can be used to effectively image and accurately characterize congenital lung malformations $[8$, 16]. However, just because you can do something does not necessarily mean that you should. One of the advantages of $\mathrm{CT}$ is that clinicians and surgeons, as well as some radiologists, while usually capable of understanding CT images and using them for guiding clinical decisions, might feel much less comfortable with MR studies. The large number of MR sequences and images obtained can be overwhelming.

Computed tomography angiography is very fast, especially with the availability of high-pitch scanning with sub-second scan duration. CT is usually easy to schedule, cheaper than 
Fig. 7 Evaluation of suspected mediastinal mass in an 11-monthold boy with an incidental mass on chest radiograph obtained for cough. a Anteroposterior chest radiograph shows a right superior rounded mediastinal density (arrow), suspected as possible neuroblastoma. b Axial T2weighted MRI for further evaluation shows a rounded T2hyperintense middle mediastinal lesion suggestive of a fluid-filled partially septated cyst (arrow). c Coronal T1-weighted postcontrast MRI confirms the cystic nature of the lesion with rim enhancement and close proximity to the airway, consistent with a mediastinal bronchogenic cyst (arrow). These findings were confirmed at surgery and pathology. Images were published in [23] in Fig. 2, reprinted with permission
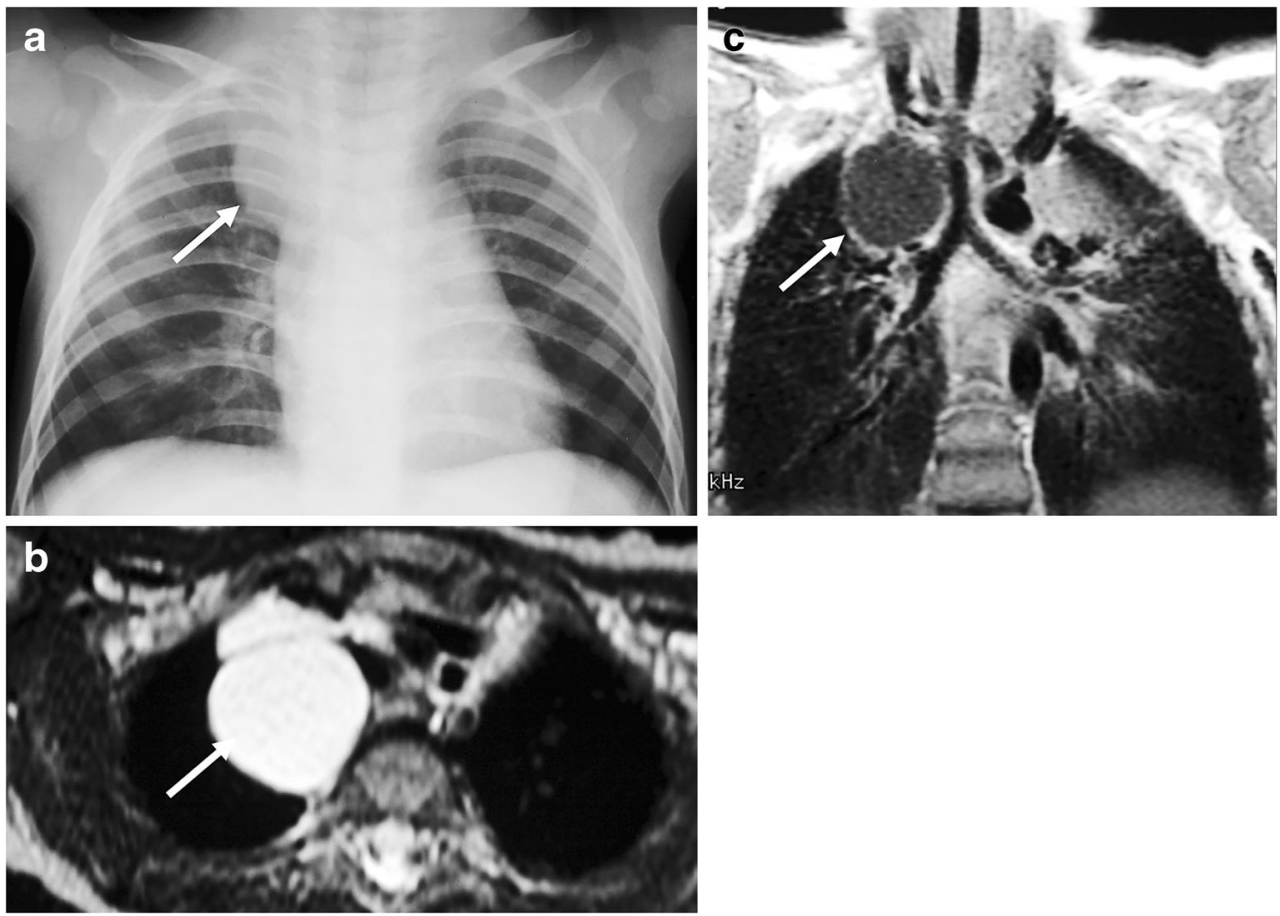

MRI, rarely requires sedation/anesthesia, and provides excellent spatial resolution and superior detail of lung parenchyma $[15,17]$. The ability to obtain very fast non-breath-hold imaging without sedation or anesthesia in most children also removes the problem of anesthesia-related atelectasis that could obscure pathology [24]. Iodinated contrast agents are not without their issues, especially in children with renal dysfunction or allergies, but they are generally considered safe.
Exposure to ionizing radiation and its possible long-term risks are of concern; however, newer equipment and imaging techniques such as low tube voltage, automatic exposure control and iterative reconstruction have allowed preservation or improvement in image quality while permitting significant reductions in CT radiation dose [19, 30]. Efforts to decrease radiation exposure usually limit $\mathrm{CT}$ angiography to a single sequence whenever possible, so contrast administration and
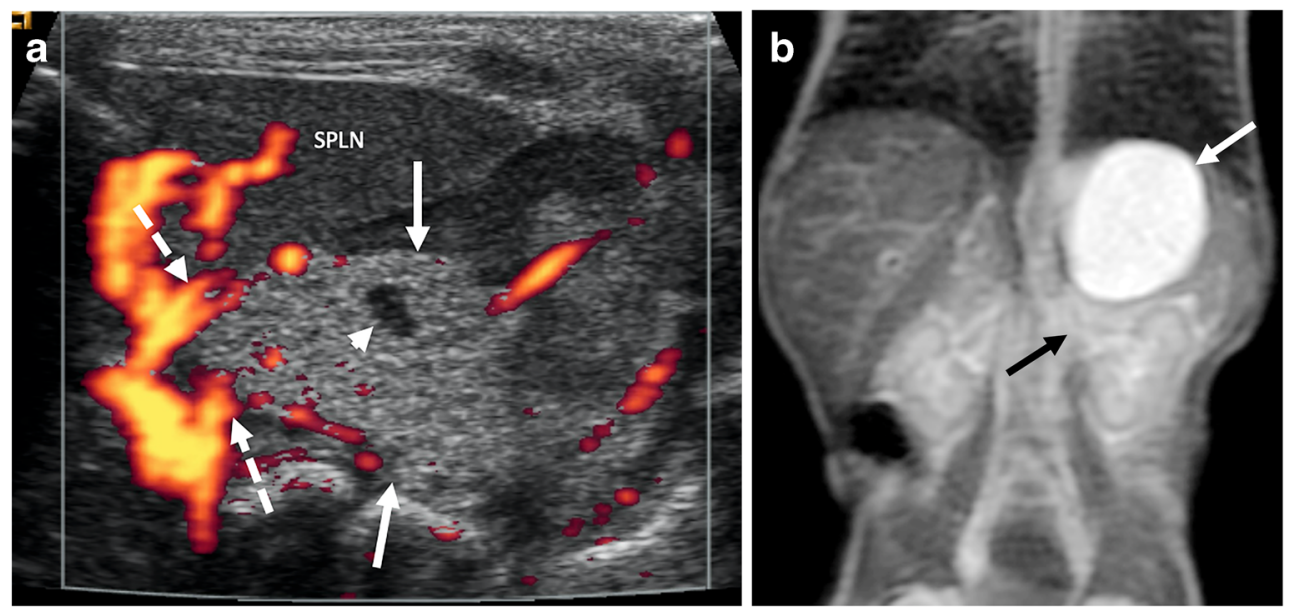

Fig. 8 Imaging following concern for neuroblastoma versus abdominal sequestration in a newborn girl with prenatal left upper abdominal mass. a Oblique US image with power Doppler shows a mostly solid echogenic mass (solid arrows) in the left upper quadrant adjacent to the spleen $(S P L N)$. There are several small internal cystic components; one is illustrated (arrowhead). Two systemic supplying vessels (dashed arrows) are identified arising from the celiac artery. b Coronal T2weighted MRI obtained as a wrap-and-feed non-sedated study without contrast agent. Image shows a large heterogeneous T2-hyperintense mass containing tiny cysts in the left upper quadrant (white arrow). The MRI clearly shows that the lesion is separate from and displacing the left adrenal gland inferiorly (black arrow). The MR appearance in combination with US findings strongly suggested an intrabdominal hybrid sequestration lesion, and these are almost always on the left side. Because of persistence and parental concern, the mass was resected at age 4 months and was confirmed to be a bronchopulmonary malformation hybrid with features of sequestration and small-cyst congenital pulmonary airway malformation 


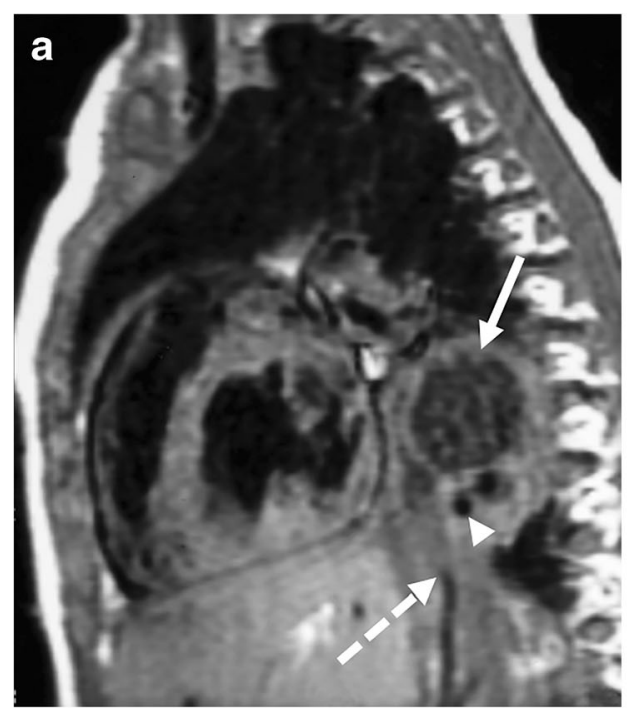

Fig. 9 Magnetic resonance imaging in a 5-month-old boy with previous repair of tracheoesophageal fistula, feeding and respiratory problems, and persistent left-lower-lobe opacity on chest radiographs. MRI was obtained to evaluate for vascular ring (no ring found). a Sagittal T1weighted dark-blood MR image in the left retrocardiac region shows a rounded complex mass in the medial left lower lobe with cystic fluid (solid arrow) and inferior more solid components. In addition, there are smaller round and very dark areas (also T2-dark, not shown) suggestive of air-filled cysts (arrowhead). An aberrant systemic artery approached

scan timing are very important. When an arterial-phase study is obtained, venous structures, especially systemic veins, might be poorly opacified such that venous anomalies and abnormal systemic venous drainage might be missed.

It is difficult to give a specific blanket recommendation as to which imaging modality to use when. Rather, the choice of

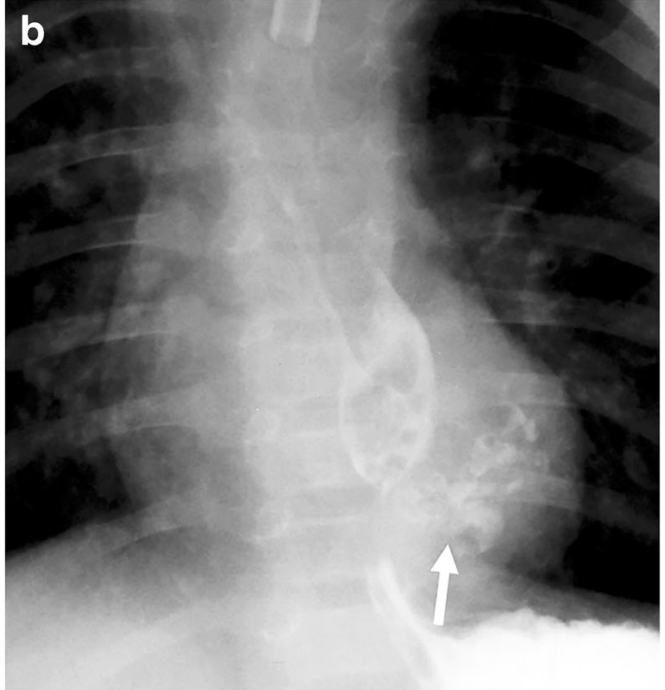

the mass from the abdomen (dashed arrow). These findings suggested a hybrid sequestration lesion. The presence of air within the mostly nonaerated lesion suggested the possibility of an aberrant connection to the gastrointestinal tract. b Contrast esophagram, anteroposterior view, demonstrates contrast filling of branching dilated airways (arrow) within the left-lower-lobe lesion, confirming an esophageal bronchus connecting to the hybrid bronchopulmonary malformation. Images were published in [1] in Fig. 5. Reprinted with permission from Springer Nature

imaging should be tailored to the individual patient, accounting for clinical circumstances, type, size and location of the lesion, parental/clinician preferences, need for and risks of sedation, imaging equipment and expertise available, and whether additional or follow-up imaging will be required in the future. With a shift to conservative management of smaller

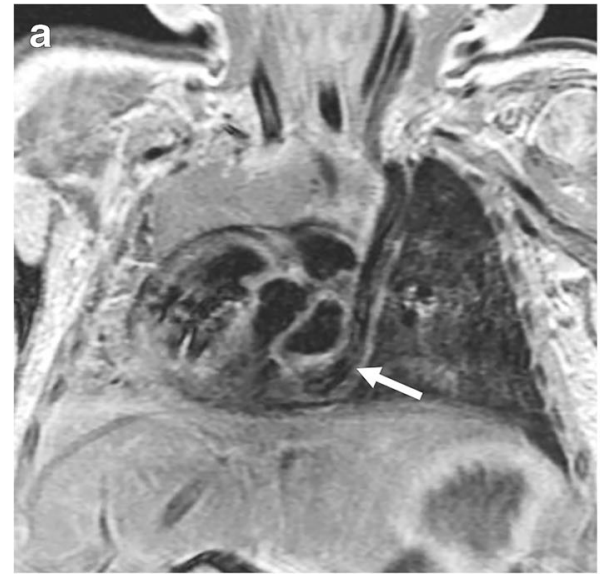

Fig. 10 Imaging in a 10-day-old boy with respiratory distress and small right lung, with a poor-quality outside CT suggesting complex scimitar syndrome. a Coronal T1-weighted spin-echo MRI shows a cardiomediastinal shift to the right with a small right lung. Left superior vena cava drains to the coronary sinus (arrow). b T1-weighted darkblood coronal MR slice, more posterior than (a), confirms a right scimitar vein (solid arrow) draining anomalously to the inferior vena cava. Multiple additional anomalies were present, including: extralobar pulmonary sequestration in a nonaerated horseshoe lung component

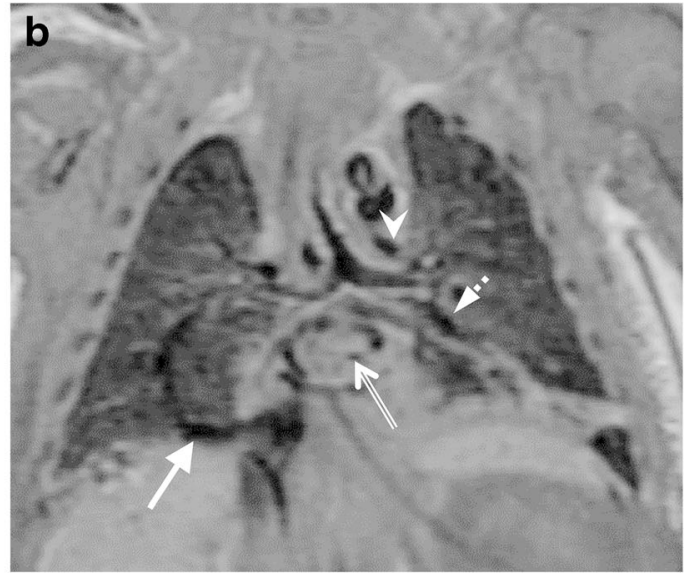

overlying the spine (open arrow); systemic arterial supply to the sequestration from the abdomen with multiple large tortuous vessels in the lesion; partial pulmonary sling with a normal left upper pulmonary artery (arrowhead) and a left lower pulmonary artery arising from the right pulmonary artery (dotted arrow); additionally, there is long-segment tracheal stenosis with a low T-shape carina, a typical configuration associated with pulmonary artery sling. Figure $10 \mathrm{~b}$ was published in [1] in Fig. 11. Reprinted with permission from Springer Nature 


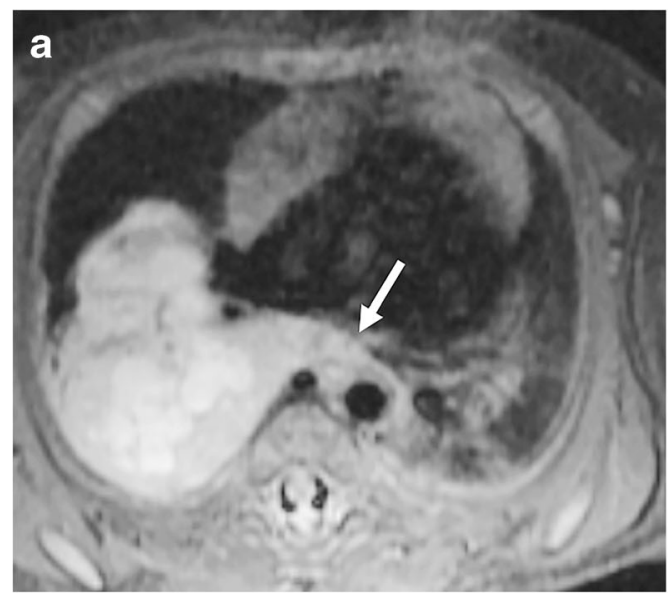

Fig. 11 Imaging in a 2-month-old boy presenting with a cough and fever; he had no chest abnormality seen prenatally. There was a rounded perihilar mass on chest radiograph (not shown), and suboptimal CT suggested differential diagnoses of necrotizing pneumonia or infected congenital pulmonary airway malformation (CPAM) versus neoplasm. a Axial short tau inversion recovery MR image shows a large right lung mass with heterogeneous T2-hyper- and hypointense solid as well as T2hyperintense fluid-filled cystic components involving the posterior right lung and extending to the mediastinum (arrow) adjacent to the aorta. b Coronal T1-W post-contrast MR shows a right-side perihilar, subcarinal and lower lobe mass with heterogeneously enhancing solid and nonenhancing cystic components extending around the hilum and right

asymptomatic bronchopulmonary malformations, MRI might be helpful for long-term monitoring of these lesions [5].

Whichever imaging modality is chosen, it is important that a high-quality contrast-enhanced (typically angiographic) study be obtained, at least initially, and that the dictated imaging report contain as complete a description as possible of the lesion. Figure 12 is the reporting template that I use for describing bronchopulmonary malformation lesions. The report should include location and size; a complete description of internal features including whether the lesion is aerated or

\section{Description of bronchopulmonary} malformation lesion

- Location:

- Size: $\mathrm{cm} \times \mathrm{cm} \times \mathrm{cm} \times$ Volume: $\mathrm{mL}$ Features:

\section{Aeration:}

2. Overinflated lung:

3. Macroscopic cyst(s):

4. Mucoid impaction:

5. Vascular:

- Arterial supply:

- Venous drainage:

\section{Lobar fissures}

7. Other

\section{Most likely type of BPM}

Fig. 12 Reporting template for bronchopulmonary malformations (BPM)

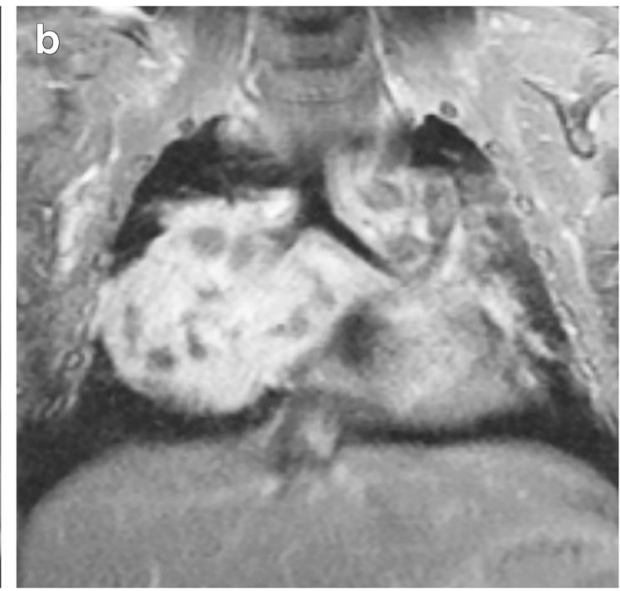

bronchi. Infected CPAM was considered unlikely because there was no prenatal history and the nonaerated lesion extended around the bronchi and to the mediastinum. Pleuropulmonary blastoma (PPB) was considered the more likely diagnosis; this would be type 2 (solid/ cystic), although almost all PPBs in infancy are type 1 (cystic). Right pneumonectomy was required for complete surgical resection. Initial pathological diagnosis was PPB type 2; subsequent external pathology consultation led to a change in diagnosis to peribronchial myofibroblastic tumor, a rare benign mesenchymal neoplasm found in late prenatal or perinatal ages. This is often a large central tumor that surrounds and entraps airways, and malformed cartilage plates are a prominent histological feature [21]

airless, the presence of overinflated lung, macroscopic cysts, mucoid impaction, and the arterial supply and venous drainage. It is also helpful to the surgeons to comment on whether the lobar fissures of the affected lung are present and complete because thoracoscopic removal is much more difficult with incomplete fissures. Other associated or incidental findings including diaphragmatic, airway, gastrointestinal and skeletal anomalies should be mentioned in addition to the general chest dictation template. The report impression can suggest the most likely specific lesion diagnoses, but that is less important than a complete description because there is often overlap among lesions [26].

\section{Conclusion}

Magnetic resonance and CT are both effective modalities for postnatal imaging of congenital lung lesions. They both have advantages and disadvantages. The choice of imaging modality is probably best individualized for the circumstances, preferences and abilities of those involved in patient care and imaging.

\section{Declarations}

Conflicts of interest None 


\section{References}

1. Newman B (2006) Congenital bronchopulmonary foregut malformations: concepts and controversies. Pediatr Radiol 36: 773-791

2. Langston C (2003) New concepts in the pathology of congenital lung malformations. Semin Pediatr Surg 12:17-37

3. Kunisaki SM, Fauza DO, Nemes LP et al (2006) Bronchial atresia: the hidden pathology within a spectrum of prenatally diagnosed lung masses. J Pediatr Surg 41:61-65

4. Riedlinger WF, Vargas SO, Jennings RW et al (2006) Bronchial atresia is common to extralobar sequestration, intralobar sequestration, congenital cystic adenomatoid malformation, and lobar emphysema. Pediatr Dev Pathol 9:361-373

5. Stanton M (2015) The argument for a non-operative approach to asymptomatic lung lesions. Semin Pediatr Surg 24:183-186

6. Cook J, Chitty LS, De Coppi P et al (2017) The natural history of prenatally diagnosed congenital cystic lung lesions: long-term follow-up of 119 cases. Arch Dis Child 102:798-803

7. Barth RA (2012) Imaging of fetal chest masses. Pediatr Radiol 42: S62-S73

8. Zirpoli S, Munari AM, Primolevo A et al (2019) Agreement between magnetic resonance imaging and computed tomography in the postnatal evaluation of congenital lung malformations: a pilot study. Eur Radiol 29:4544-4554

9. Waelti SL, Garel L, Soglio DD et al (2017) Neonatal congenital lung tumors - the importance of mid-second-trimester ultrasound as a diagnostic clue. Pediatr Radiol 47:1766-1775

10. Baird R, Puligandla PS, Laberge JM (2014) Congenital lung malformations: informing best practice. Semin Pediatr Surg 23: 270-277

11. Pacharn P, Kline-Fath B, Calvo-Garcia M et al (2013) Congenital lung lesions: prenatal MRI and postnatal findings. Pediatr Radiol 43:1136-1143

12. Zucker EJ, Epelman M, Newman B (2015) Perinatal thoracic mass lesions: pre- and postnatal imaging. Semin Ultrasound CT MR 36: 501-521

13. Kunisaki SM, Ehrenberg-Buchner S, Dillman JR et al (2015) Vanishing fetal lung malformations: prenatal sonographic characteristics and postnatal outcomes. J Pediatr Surg 50:978-982

14. Newman B, Caplan J (2014) Cystic lung lesions in newborns and young children: differential considerations and imaging. Semin Ultrasound CT MR 35:571-587

15. Thacker PG, Schooler GR, Caplan MJ, Lee EY (2015) Developmental lung malformations in children: recent advances in imaging techniques, classification system, and imaging findings. J Thorac Imaging 30:29-43

16. Kellenberger CJ, Amaxopoulou C, Moehrlen U et al (2020) Structural and perfusion magnetic resonance imaging of congenital lung malformations. Pediatr Radiol 50:1083-1094
17. Epelman M, Kreiger PA, Servaes S et al (2010) Current imaging of prenatally diagnosed congenital lung lesions. Semin Ultrasound CT MR 31:141-157

18. Feinberg A, Hall NJ, Williams GM et al (2016) Can congenital pulmonary airway malformation be distinguished from type I pleuropulmonary blastoma based on clinical and radiological features? J Pediatr Surg 51:33-37

19. Haggerty JE, Smith EA, Kunisaki SM, Dillman JR (2015) CT imaging of congenital lung lesions: effect of iterative reconstruction on diagnostic performance and radiation dose. Pediatr Radiol 45: 989-997

20. Owada K, Miyazaki O, Matsuoka K et al (2015) Unusual signal intensity of congenital pulmonary airway malformation on fetal magnetic resonance imaging. Pediatr Radiol 45:763-766

21. Brock KE, Wall J, Esquivel M et al (2015) Congenital peribronchial myofibroblastic tumor: case report of an asymptomatic infant with a rapidly enlarging pulmonary mass and review of the literature. Ann Clin Lab Sci 45:83-89

22. Victoria T, Srinivasan AS, Pogoriler J et al (2018) The rare solid fetal lung lesion with T2-hypointense components: prenatal imaging findings with postnatal pathological correlation. Pediatr Radiol 48:1556-1566

23. Newman B (2011) Thoracic neoplasms in children. Radiol Clin N Am 49:633-664

24. Kino A, Zucker EJ, Honkanen A et al (2019) Ultrafast pediatric chest computed tomography: comparison of free-breathing vs. breath-hold imaging with and without anesthesia in young children. Pediatr Radiol 49:301-307

25. Kline-Fath BM, Calvo-Garcia MA, O'Hara SM et al (2007) Twintwin transfusion syndrome: cerebral ischemia is not the only fetal MR imaging finding. Pediatr Radiol 37:47-56

26. Epelman M, Lee E, Newman B (2019) Congenital lung anomalies. In: Coley BD (ed) Caffey's pediatric diagnostic imaging, 13th edn. Elsevier, Amsterdam, p 495

27. Baez JC, Ciet P, Mulkern R et al (2015) Pediatric chest MR imaging: lung and airways. Magn Reson Imaging Clin N Am 23:337349

28. Zucker EJ, Cheng JY, Haldipur A et al (2018) Free-breathing pediatric chest MRI: performance of self-navigated golden-angle ordered conical ultrashort echo time acquisition. J Magn Reson Imaging 47:200-209

29. Cravero JP, Callahan MJ (2017) The radiological home: pediatric anesthesiologist's role in risk assessment for imaging procedures. Paediatr Anaesth 27:878-879

30. Tucker EW, Jain SK, Mahesh M (2017) Balancing the risks of radiation and anesthesia in pediatric patients. J Am Coll Radiol 14:1459-1461

Publisher's note Springer Nature remains neutral with regard to jurisdictional claims in published maps and institutional affiliations. 